\title{
Nasal Profile Changes Following Anterior Maxillary Segmental Osteotomy: A Lateral Cephalometric Study
}

\author{
Rastogi Komal $^{1,2,4,}$ - Pai K. Deepak ${ }^{1,3} \cdot$ Choonthar M. Muralee $^{1}$ M. S. Ravi ${ }^{1}$
}

Received: 28 October 2014/Accepted: 15 April 2015/Published online: 8 May 2015

(C) The Association of Oral and Maxillofacial Surgeons of India 2015

\begin{abstract}
Purpose Orthognathic surgery in reference to the maxilla attempts to correct underlying skeletal deformities and improve function. Consequently it has the potential to significantly alter the central esthetic unit of the face, the nasolabial region. In order to evaluate the nasal morphological changes which would result following anterior maxillary segmental osteotomy (superior and posterior repositioning); four angles namely, nasolabial, nasal tip projection, columellar labial and supra tip break angle were evaluated.

Method and materials In ten selected subjects who have undergone anterior maxillary segmental osteotomy with superior and posterior repositioning, pre (T1) and post operative (T2) lateral cephalometric parameters pertaining to the four angles were analysed using Wilcoxon signed rank test.
\end{abstract}

Rastogi Komal

dr.komalrastogi@gmail.com

Pai K. Deepak

drdeepakpai@yahoo.com

Choonthar M. Muralee

drmuraleemohan@gmail.com

M. S. Ravi

drmsravi@gmail.com

1 A.B.Shetty Memorial Institute of Dental Sciences, Mangalore, India

2 Present Address: MR Ambedkar Dental College, Bangalore, India

3 Present Address: A.J.Shetty Institute of Dental Sciences, Mangalore, India

4 House No 13, First Cross Bassappa Road, Shantinagar, Bangalore, India
Results A significant increase in nasolabial angle and mild changes in nasal tip projection, columellar labial angle and supra tip break angle were observed.

Conclusion The results of this study emphasize the need for the pre surgical evaluation of nasal morphology in every individual planned for anterior maxillary segmental osteotomy.

Keywords Anterior maxillary segmental osteotomy . Nasal profile $\cdot$ Superior and posterior repositioning · Lateral cephalometrics

\section{Introduction}

Orthognathic surgery in reference to the maxilla attempts to correct underlying skeletal deformities and improve function. Consequently it has the potential to significantly alter the central esthetic unit of the face, the nasolabial region. The nose is a keystone of facial esthetics and thus is of central importance in planning and execution of orthognathic surgery $[1,2]$. A key to achieving a good functional as well as esthetic result involves both comprehensive surgical planning and an understanding of the effects that orthognathic surgery of the maxilla will have on the soft tissues within the nasolabial region. This is why the standard or classic lateral cephalometric skeletal analyses need to be augmented by the addition of soft-tissue evaluations.

Although a number of studies pertaining to soft tissue changes of the face with the maxillary procedure can be found in the orthodontic and oral surgery literature, information is limited when detailed evaluation of changes specific to nasal morphology is desired [3-5]. This incited need for the study. 


\section{Materials and Methods}

\section{Ethical Approval}

Ethical approval was obtained from the Institutional Ethical clearance committee.

Ten subjects, six females and four males between 18 and 25 years of age diagnosed with Skeletal class II malocclusion or Bimaxillary protusion requiring anterior maxillary segmental osteotomy (superior and posterior vector movement of maxilla) were selected for the study. None of these patients had any adjunctive nasal surgery like, septoplasty or rhinoplasty being performed in conjunction with the anterior segmental osteotomy. Subjects with associated syndromes, cleft lip and palate deformity or facial asymmetry were excluded. After obtaining written informed consent from each individual, lateral cephalogram and other diagnostic records were made. All the ten individuals selected for the study underwent pre surgical and post surgical orthodontic treatment.

Out of the ten subjects, six of them underwent anterior segmental osteotomy in maxilla for superior and posterior repositioning and advancement genioplasty in mandible. Two patients underwent anterior segmental osteotomy in the maxilla and anterior sub apical osteotomy in the mandible. Remaining two patients were treated only with anterior segmental osteotomy in maxilla as a single procedure.

\section{Methodology}

The nasal profile was evaluated by lateral cephalogram analysis in two stages as follows:

$\mathrm{T}_{1} \quad$ Pre Surgical (2-3 weeks before surgery)

$\mathrm{T}_{2} \quad$ Post Surgical (6 months after surgery)

Lateral cephalograms were obtained as per standard criteria, in centric occlusion with lips in repose using the PlanMeca 2002 Proline Cephalometric unit (HelsinkiFinland) at the same target to film and subject to film distances. All the lateral cephalograms were hand traced by single operator using $0.003^{\prime \prime}$ acetate tracing sheet and $0.5 \mathrm{~mm}$ lead pencil.

Two cephalometric landmarks: Sella and Nasion were identified and utilised to construct the two reference lines as follows:

- Nasion horizontal $(\mathrm{NH})$ : A horizontal reference line constructed at $7^{\circ}$ above sellanasionatnasion.

- Nasion vertical (NV): A vertical reference line constructed at $90^{\circ}$ to $\mathrm{NH}$.
Following soft tissue points that were identified for evaluation of nasal profile:

- Supra nasal tip (SNt): the most anterosuperior point on the outline of the soft tissue nose, located with a tangent from nasion.

- Anterior nasal tip (Ant): the most anterior point on the outline of the soft tissue nose, located as the most anterior perpendicular to nasal vertical.

- Collumella point $(\mathrm{Cm})$ : the most anterior point on the columella of the nose.

- LabraleSuperius (Ls): The most anterior point on the upper lip.

- Subnasale (Sn): The point at which the nasal septum merges with the upper cutaneous lip in the mid saggital plane.

Following four angles were measured up to accuracy of $0.5^{\circ}$ and evaluated:

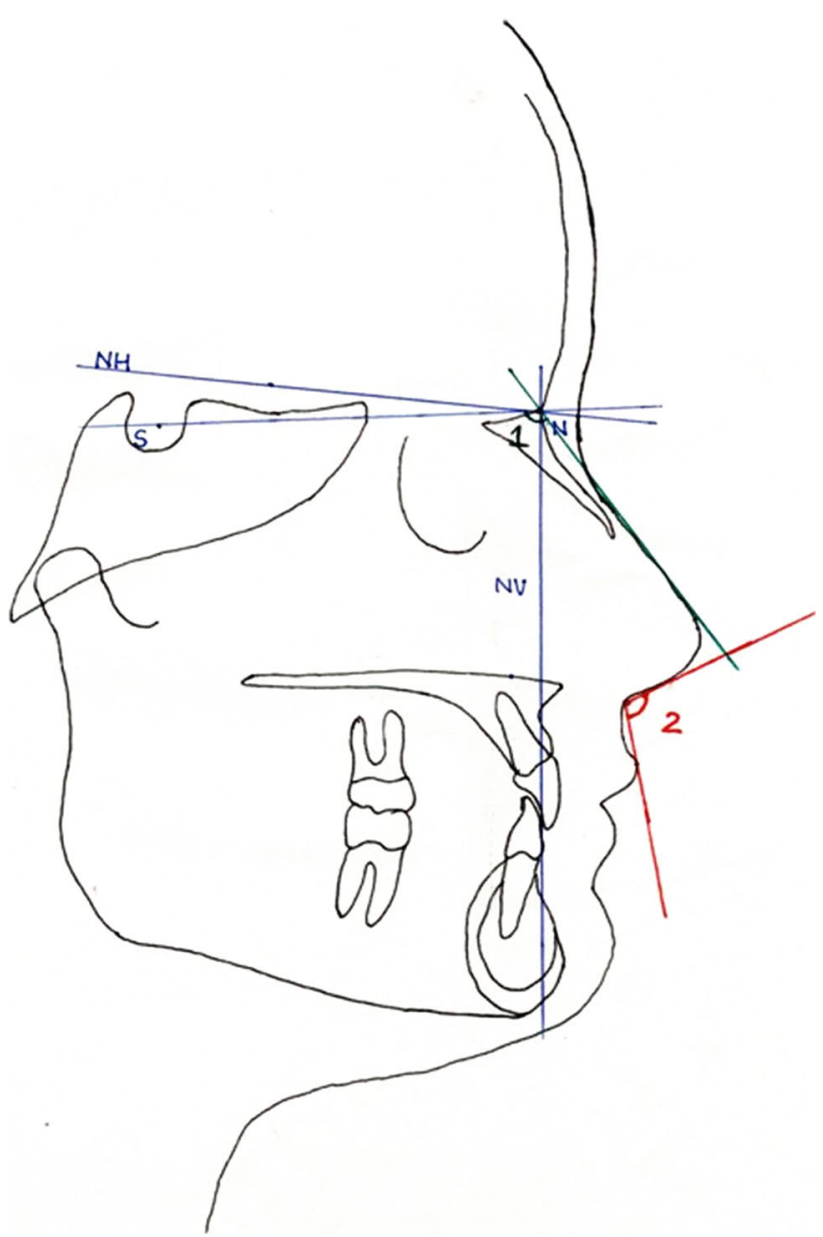

Fig. 1 Nasal tip projection angle (1), Nasolabial angle (2). $N$ nasion, $S$ sella, $N H$ nasion horizontal, $N V$ nasion vertical 
- Nasal tip projection angle (NTPA): measured from the horizontal line $(\mathrm{NH})$ to the most anterior superior point on the nose [Fig. 1].

- Nasolabial angle (NLA): measured as the angle formed by the nasal columella and labial philtrum. (Cm-Sn-Ls) [Fig. 1].

- Collumellar labial angle (CA): measured from the vertical reference line to collumellar tangent [Fig. 2].

- Supra Tip Break Angle (STBA): measured from the nasal dorsum tangent to the supra tip tangent [Fig. 2].

The post surgical $\left(\mathrm{T}_{2}\right)$ tracing was superimposed on pre surgical $\left(\mathrm{T}_{1}\right)$ tracing using anterior cranial base structures and two reference lines [Fig. 3].

\section{Surgical Technique}

An incision was placed in labial vestibule, $5 \mathrm{~mm}$ above the attached mucosa. Maxillary first premolars were extracted intra operatively. Along with subperiosteal reflection of the

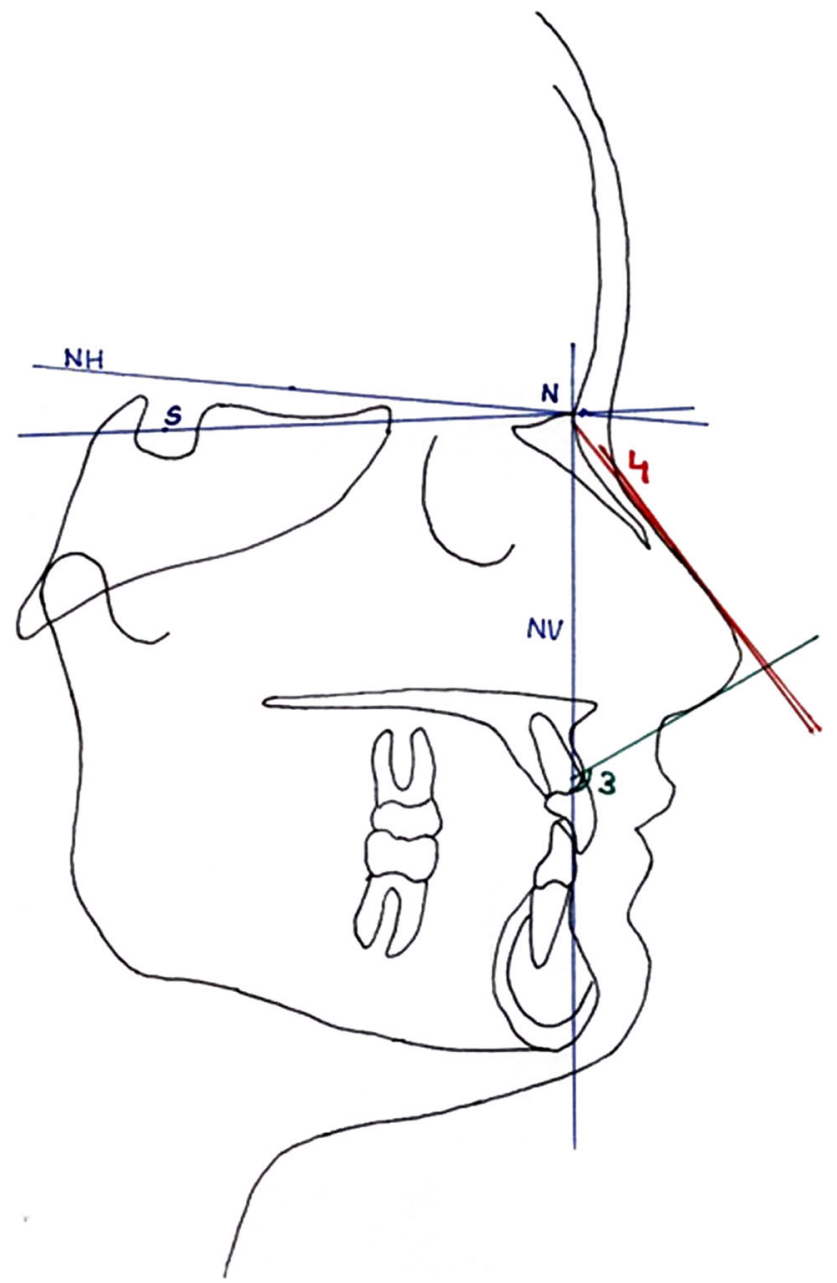

Fig. 2 Columellar labial angle (3), Supra tip break angle (4). $N$ nasion, $S$ sella, $N H$ nasion horizontal, $N V$ nasion vertical

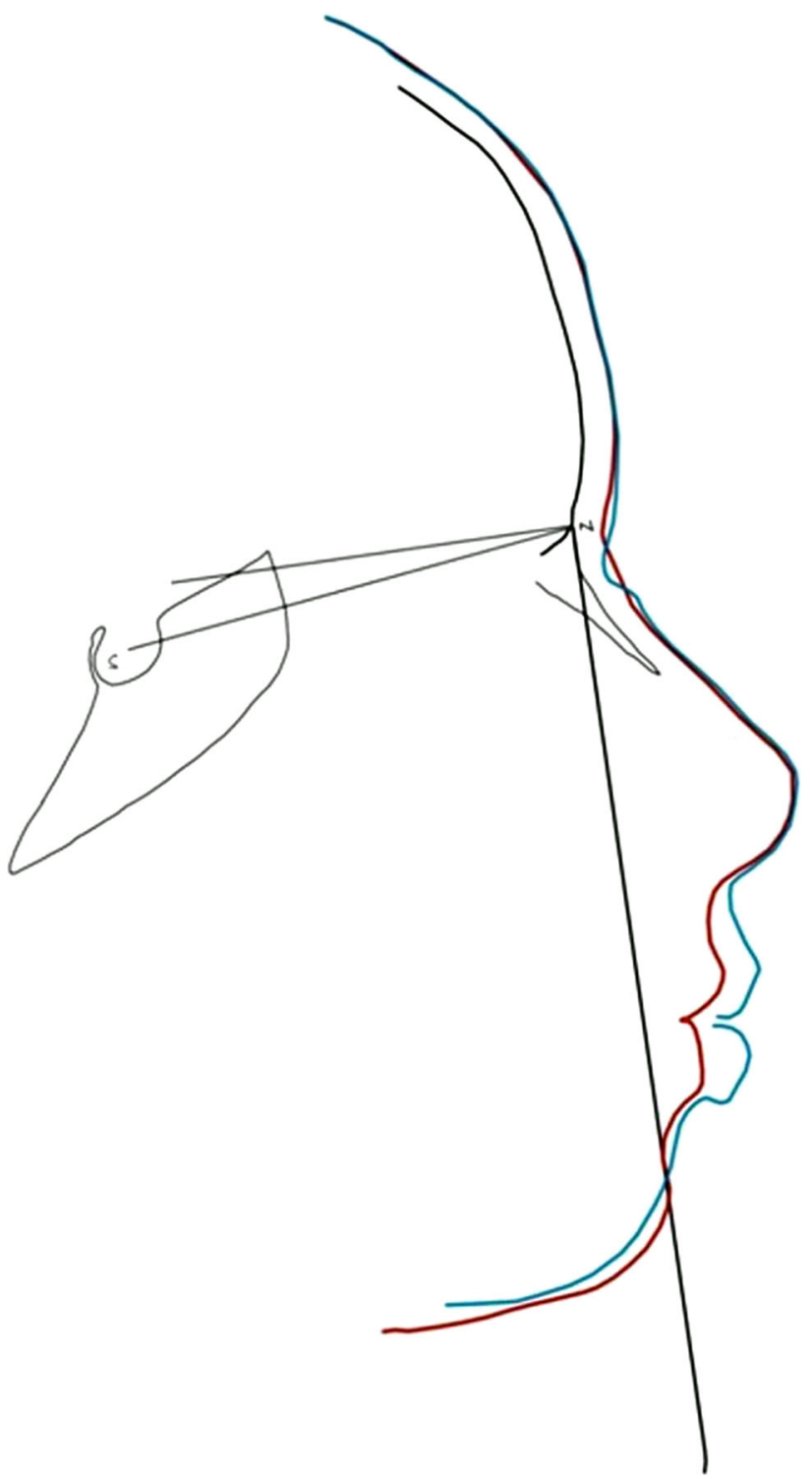

Fig. 3 Pre and post surgical superimposition tracing. Blue pre operative soft tissue outline (T1), Red post operative soft tissue outline (T2)

labial mucosa, palatal tunnelling and meticulous reflection of nasal mucosa was performed. The horizontal osteotomy cuts were placed from the pyriform rim extending laterally to join the vertical osteotomy cuts through the extraction sockets bilaterally and transpalatal osteotomy was completed. Nasal septum was trimmed by $2-3 \mathrm{~mm}$ to suit the clinical requirement and prevent its buckling. Although anterior nasal spine was left intact, yet any sharp anterior nasal spine was trimmed. The osteotomized segments were stabilized with either two mm thickness L plates or four hole mini plates.

In all the ten subjects, superior repositioning achieved was with a mean value of four $\mathrm{mm}$ and posterior repositioning was four and half $\mathrm{mm}$. 
Wound site was closed with 3-0 Vicryl (Polygalactin 910) in V-Y pattern to achieve increase in lip length; however alar cinch sutures were not placed.

A significant improvement in nasal and facial profile was achieved in all ten patients [Figs. 4, 5, 6, 7].

\section{Statistical Analysis}

The data collected were subjected to Paired $t$ test. A $p$ value of less than 0.05 was considered to be significant and any value more than 0.05 was considered not significant [Figs. 8, 9, 10, 11].

\section{Results}

The four angles NLA, NTPA, CA and STBA were evaluated and a comparison was done between the pre surgical (T1) and post surgical (T2) values in a group of ten patients. The statistical analysis demonstrated highly significant post operative changes in NLA with a $p$ value of

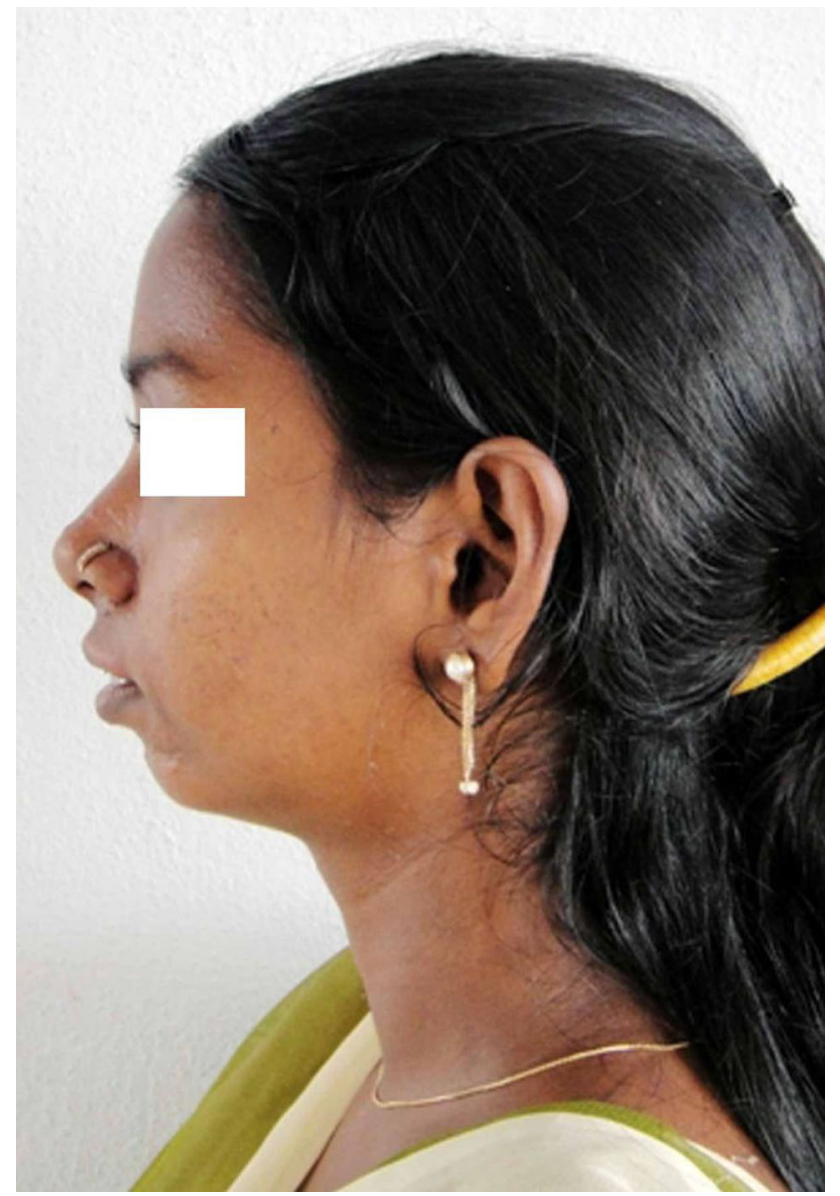

Fig. 4 Pre surgical lateral profile photograph

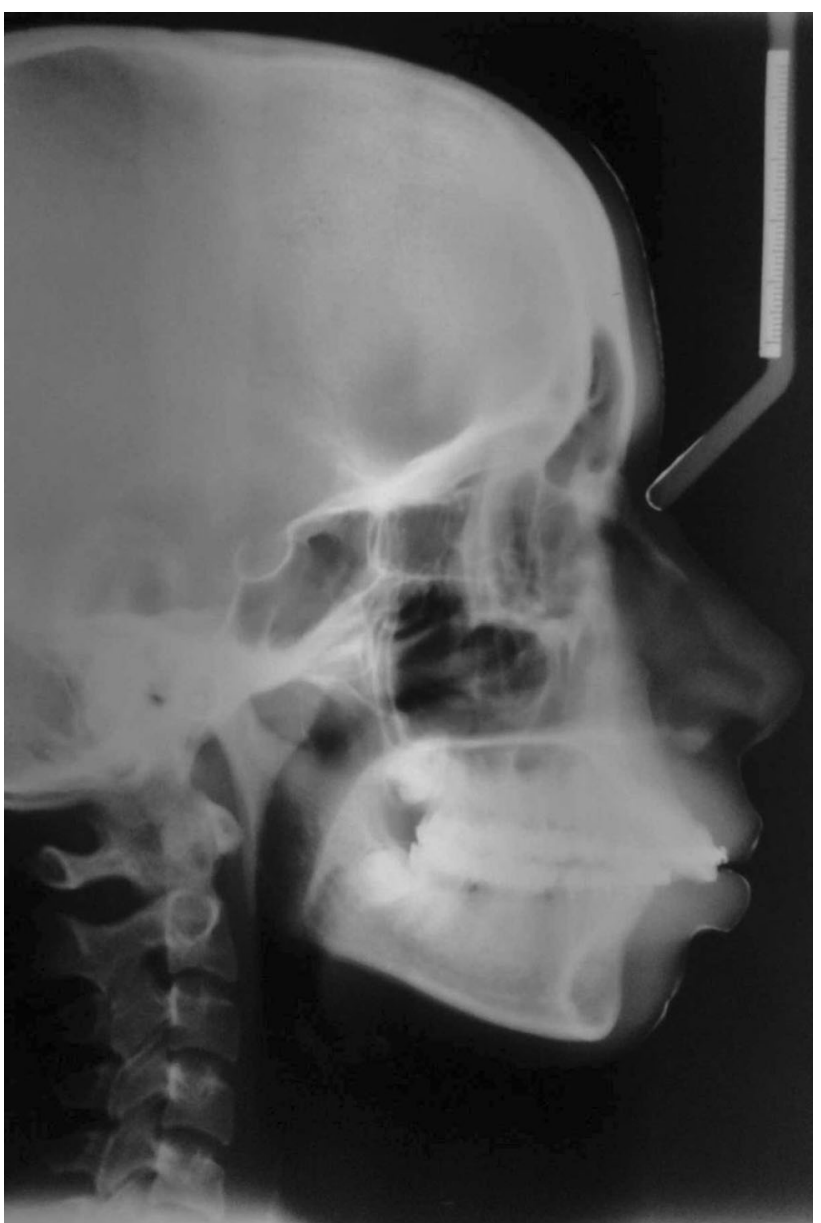

Fig. 5 Pre surgical lateral cephalogram (T1)

0.006. The changes in other three angles did not show significant changes post surgically (Tables 1, 2, 3, 4).

\section{Discussion}

Maxillary surgical procedures have shown significant alterations in the nasolabial region, which are considered to be the central esthetic unit of the face. These changes could be widening of the base of the nose and associated flattening and thinning of the upper lip, especially noticeable as loss of the visible vermilion border. Many factors like, the direction of the skeletal movement, the handling of the soft tissues, the skin thickness and the pre existing nasal structure demonstrate considerable effect over changes in nasal tip structure and dorsum. Nasal morphological changes are secondary to alterations in the regional anatomy associated with surgical repositioning. The consequential effect of the surgery is seen in the overlying skin and subcutaneous tissue as changes in the previously identified external landmarks [6]. 


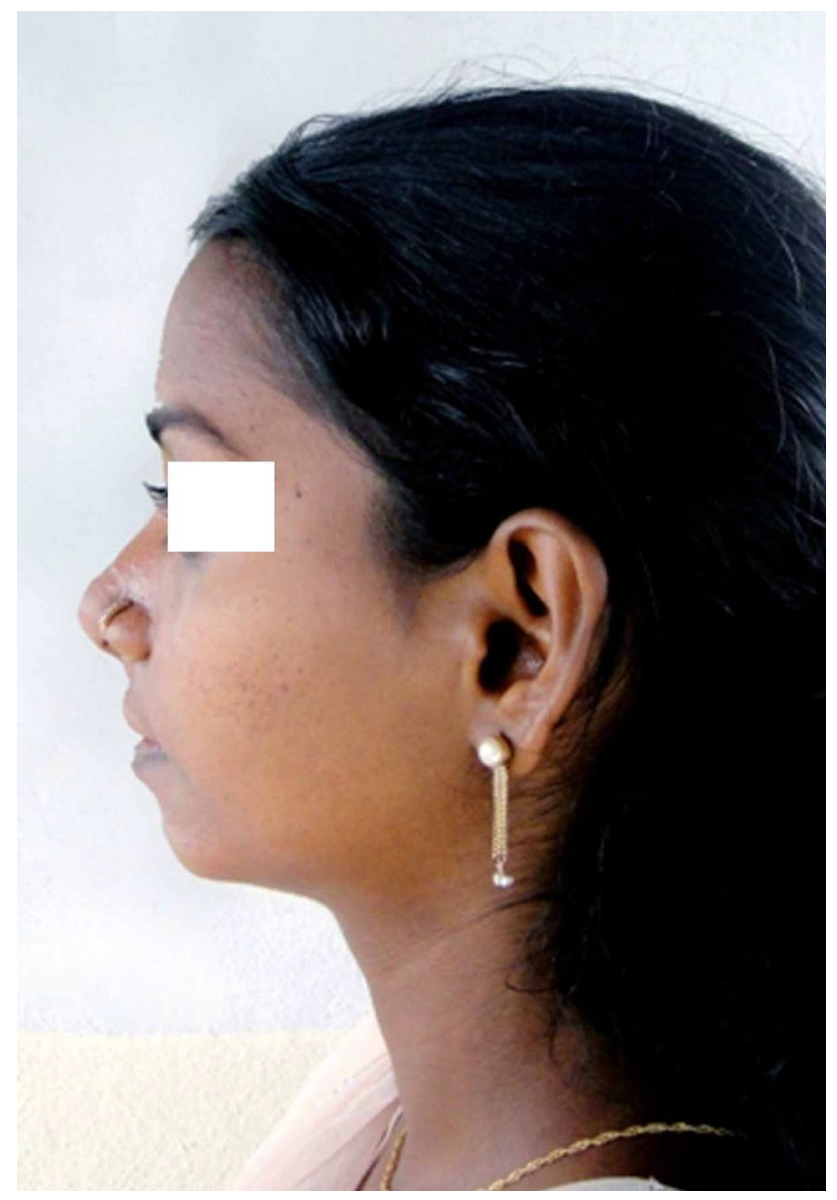

Fig. 6 Post surgical lateral profile photograph

Various studies in literature have been conducted for the assessment of soft tissue changes in response to anterior segmental maxillary osteotomy using lateral cephalograms. A study by Jayaratne et al. [7] done to evaluate the facial soft tissue response to anterior maxillary osteotomies was performed based on the electronic databases including 11 studies. The review of these 11 studies indicated a reduction of the labial prominence with an increase in the nasolabial angle ranging from $8.9^{\circ}$ to $18^{\circ}$. The magnitude of the reported soft tissue changes and their ratios corresponding to the osseous movements varied among studies.

Park and Hwang [8] in their study on a group of 30 patients have stated an increase of the nasolabial angle ranging from $94.96^{\circ} \pm 9.67^{\circ}$ to $109.03^{\circ} \pm 9.08^{\circ}$.

A pilot study conducted on 20 adult patients to investigate and predict soft tissue changes in the forehead, nose, lips and chin in association with anterior maxillary osteotomy have demonstrated backward displacement of the subnasale and the upper and lower lips [9].

In a case report Natao Suda et al. [10] have stated that the anterior maxillary osteotomy causes an increase in value of nasolabial angle with a mean of $+3.5^{\circ}$ to $11.0^{\circ}$.

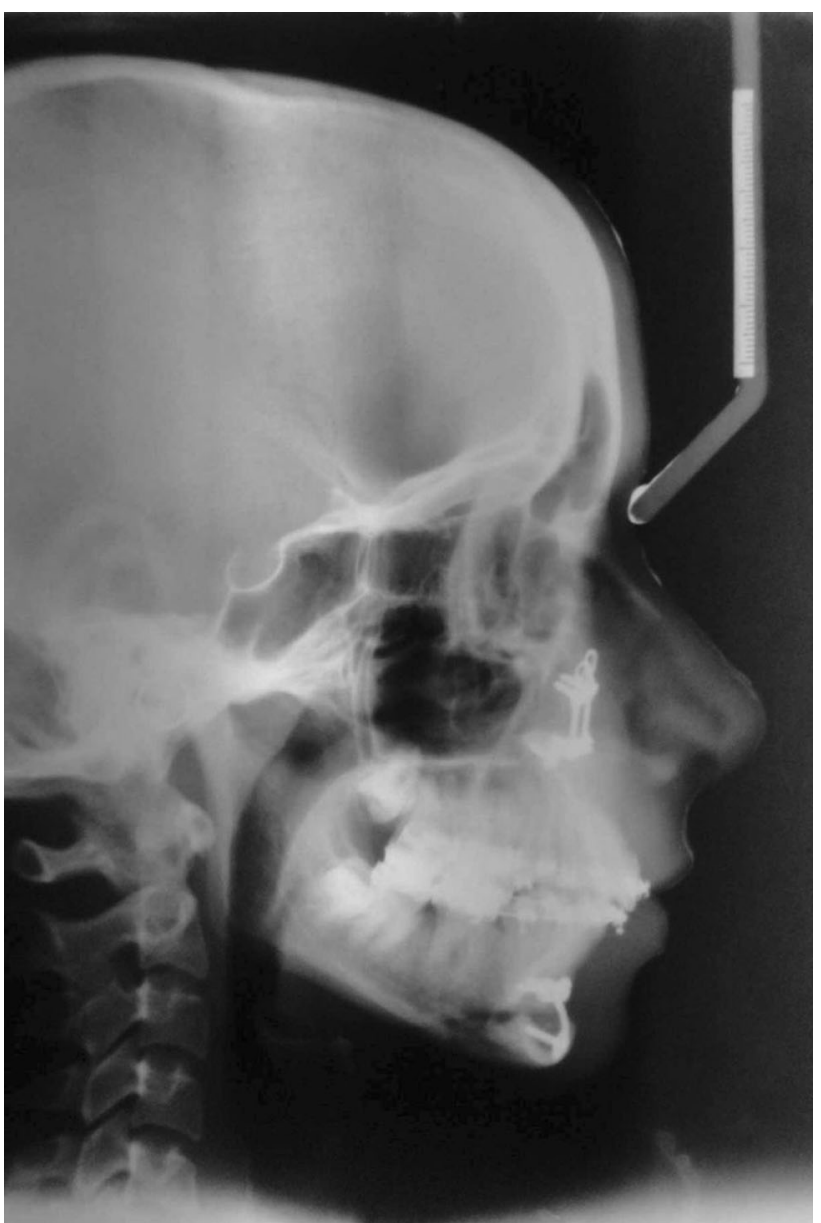

Fig. 7 Post surgical lateral cephalogram (T2)

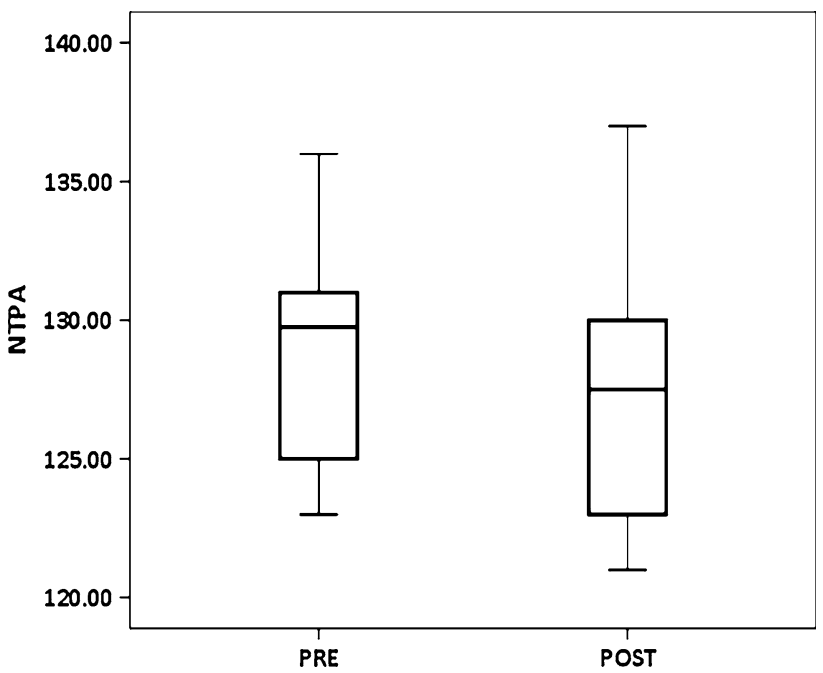

Fig. 8 Pre and post surgical NTPA (Box plots showing minimum, maximum, median \& interquartile range)

In view of the nasal changes pertaining to maxillary surgical procedures, the present study has been carried out 


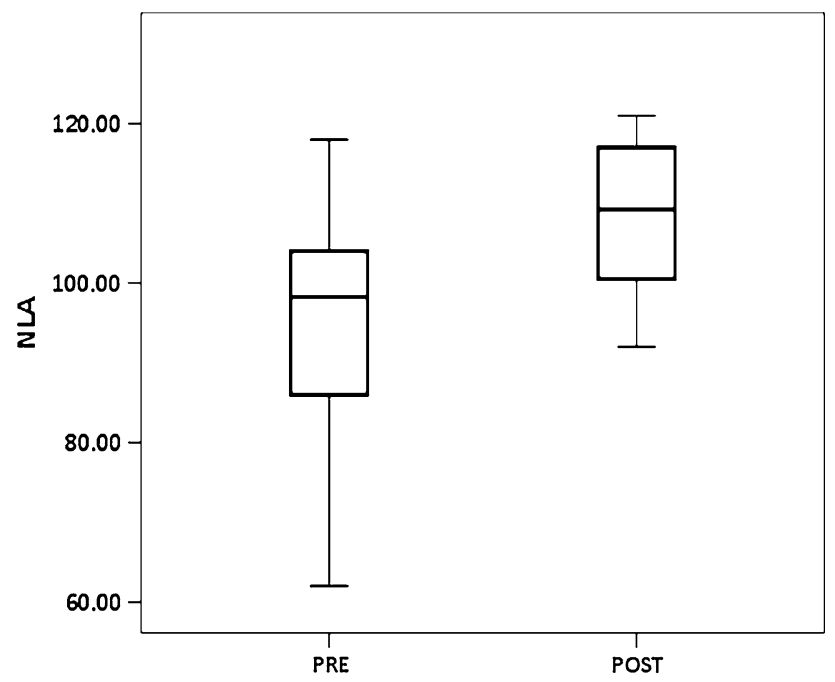

Fig. 9 Pre and post surgical NLA (Box plots showing minimum, maximum, median $\&$ interquartile range)

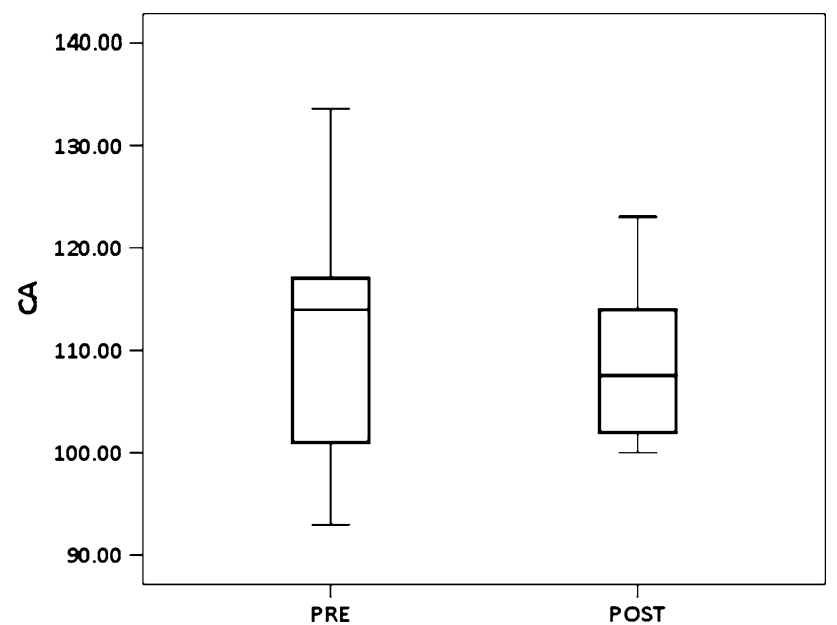

Fig. 10 Pre and post surgical CA (Box plots showing minimum, maximum, median \& interquartile range)

to evaluate nasal profile changes following superior and posterior maxillary impaction osteotomy. Pre surgical (T1) and Post surgical (T2) lateral cephalogram of each patient was evaluated. Postsurgical cephalogram was made after 6 months of time interval as suggested by many authors [11-13], in order to allow soft tissue stabilization and adaptation.

All the lateral cephalograms were made using standardized cephalometric technique. The same cephalometer with the same source subject and subject film distance and patient positioning in natural head position with the teeth in centric occlusion and soft tissue in repose. On the pre surgical and post surgical cephalograms two landmarks were used namely, Sella and Nasion as their reliability was found to be high [14]. Only these two landmarks were

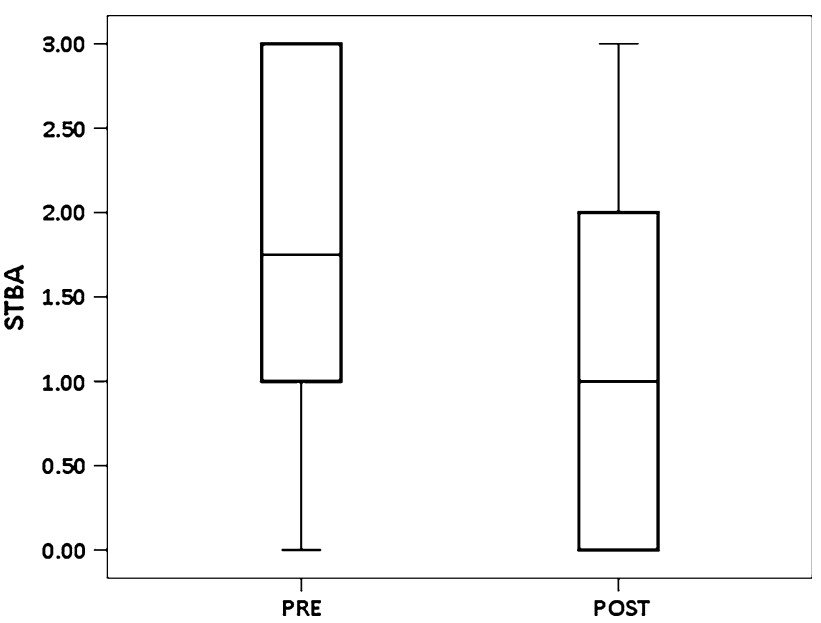

Fig. 11 Pre and post surgical STBA (Box plots showing minimum, maximum, median \& interquartile range)

required to construct the two reference lines so that high reproducibility and reliability was ensured. All additional soft tissue points were marked on the cephalograms. The post surgical tracing was then superimposed onto the pre surgical tracing (using anterior cranial base structures) and the two reference lines were transferred. The four angles NTPA, NLA, CA and STBA were evaluated in T1 and T2 and then superimposed to evaluate the changes between the values of the above four angles.

Only one angle, NLA showed significant post surgical changes with a significant $p$ value of 0.006 . The increase in nasolabial angle demonstrated a range of $3^{\circ}$ to $38.5^{\circ}$ (mean value $12.45^{\circ}$ ). The overall range of nasolabial angle post operatively in our patients was $92^{\circ}$ to $117^{\circ}$ which followed the normal value range. This increase in NLA can be attributed to movement of soft tissue point Ls and Sn. The movement of soft tissue point Ls seems to be related to maxillary incisor retraction and also associated with setback or retrusion of the anterior maxillary segment. Movement of the maxilla in a posterior vector implicates changes in upper lip morphology and changes in position of the soft tissue point Ls and $\mathrm{Sn}$ in a posterior direction. Thus, results in a significant opening of NLA (Cm-Sn-Ls). As most of the patients included in this study were females $(60 \%)$, this increase in nasolabial angle did enhance the nasal profile post operatively. The result obtained in regard to NLA were in concordance with various studies [7-10] carried out to evaluate the soft tissue changes after anterior maxillary osteotomy.

Although statistically no significant changes were seen in nasal tip projection and columella labial angle, they demonstrated a range of changes in their value by $-10^{\circ}$ to $+3^{\circ}$ and $-11^{\circ}$ to $+14^{\circ}$ respectively. These results were in agreement with many other authors $[7,9,15]$. In this study NTPA has shown a minimal decrease in its value by 
Table 1 Summary measures of pre (T1) \& post surgical (T2) NTPA

Table 2 Summary measures of pre \& post surgical NLA

\begin{tabular}{llllll}
\hline & N & Mean \pm SD & I Quartile & Median & III Quartile \\
\hline NTPA pre (T1) & 10 & $128.7 \pm 4.14$ & 124.88 & 129.75 & 131.25 \\
NTPA post (T2) & 10 & $127.6 \pm 5.01$ & 122.88 & 127.50 & 130.63 \\
\hline
\end{tabular}

Wilcoxon signed ranks test

NTPA post-NTPA pre

Z

$-.852^{\mathrm{a}}$

$p$ value

0.394 (Not significant)
Table 3 Summary measures of pre \& post surgical CA

\begin{tabular}{llrlrl}
\hline & $\mathrm{N}$ & \multicolumn{1}{c}{ Mean $\pm \mathrm{SD}$} & I Quartile & Median & III Quartile \\
\hline NLA PRE (T1) & 10 & $95.5 \pm 16.16$ & 84.25 & 98.25 & 105.50 \\
NLAPOST (T2) & 10 & $107.95 \pm 10.11$ & 98.50 & 109.25 & 117.00 \\
\hline
\end{tabular}

Wilcoxon signed ranks test

Z

NLA post-NLA pre

$p$ value

$-2.701^{\mathrm{a}}$

0.007 (Significant)

${ }^{a}$ Based on negative ranks

\begin{tabular}{llllll}
\hline & $\mathrm{N}$ & Mean $\pm \mathrm{SD}$ & I Quartile & Median & III Quartile \\
\hline CA PRE (T1) & 10 & $111.3 \pm 12.44$ & 100.00 & 114.00 & 118.50 \\
CAPOST (T2) & 10 & $108.9 \pm 8.5$ & 101.75 & 107.50 & 116.00 \\
\hline
\end{tabular}

Wilcoxon signed ranks test

\begin{tabular}{ll}
\hline & CA post-CA pre \\
$\mathrm{Z}$ & $-.868^{\mathrm{a}}$ \\
Asymp. Sig. (2-tailed) & 0.386 (Not significant)
\end{tabular}

${ }^{a}$ Based on positive ranks

\begin{tabular}{llllll}
\hline & $\mathrm{N}$ & Mean $\pm \mathrm{SD}$ & I Quartile & Median & III Quartile \\
\hline STBAPRE (T1) & 10 & $1.7 \pm 1.18$ & 0.75 & 1.75 & 3.00 \\
STBAPOST (T2) & 10 & $1.05 \pm 1.01$ & 0.00 & 1.00 & 2.00 \\
\hline
\end{tabular}

Wilcoxon signed ranks test

STBA post-STBA pre

Z

$-.872^{\mathrm{a}}$

Asymp. Sig. (2-tailed)

0.383 (Not significant)

${ }^{a}$ Based on positive ranks

a mean of $-1.1^{\circ}$ which is in contrast to a study conducted by Park et al. [8] which states a minimal increase in nasal tip projection following anterior maxillary segmental osteotomy. The change in nasal tip projection and columellar angle could not be appreciated clinically. Supra tip break angle exhibited a mean change of $-0.65^{\circ}$ which was 
statistically not significant. Further studies may be required to establish the change in supra tip break in regard to maxillary orthognathic procedures.

The result of this study does demonstrate nasal changes following anterior maxillary segmental osteotomy with a superior and posterior repositioning. The technique of Anterior Maxillary osteotomy dictates osteotomy cuts to be at the level of anterior nasal spine and also it requires minimal perinasal musculature dissection. It requires minimum retraction of the paranasal soft tissues and an intact anterior nasal spine. Also, the degree of superior impaction that can be achieved by Anterior Maxillary osteotomy is limited by the level of the osteotomy cuts. Thus, a consequent favourable nasal change is to be expected following Anterior Maxillary osteotomy.

Thus it can be concluded that the anterior segmental osteotomy in the maxilla for superior and posterior repositioning has resulted in significant opening of nasolabial angle and mild change in nasal tip projection angle.

Thus, subjects with acute nasolabial angle and poor nasal tip projection will be benefited with enhancement in their nasal profile following anterior maxillary osteotomy. Surgical goals must maximize the soft tissue esthetics. Hence, the importance of pre surgical planning with soft tissue analysis specific to nasal morphology in a patient planned for maxillary orthognathic procedures cannot be underestimated. Further long term studies are required with large number of samples and in different ethnic groups.

Acknowledgments Authors acknowledge the support extended by Prof. (Dr.) S.M. Sharma, Head of the Department of Oral and Maxillofacial Surgery and Prof.(Dr.) B. Rajendra Prasad, Dean and Principal, A.B. Shetty Memorial Institute of Dental Sciences, Mangalore. India.

\section{Conflict of interest None.}

Ethical approval Obtained from the Institutional Ethical clearance committee.

\section{References}

1. Peck GC, Michelson LN (1987) Anatomy of aesthetic surgery of the nose. Clin Plast Surg 14:737-748

2. Sheen J, Sheen A (1987) Aesthetic rhinoplasty, vol I. Esthetics and techniques. St. Louis, CV Mosby

3. Engel GA, Quan RE, Chaconas SJ (1979) Soft tissue change as a result of maxillary surgery: a preliminary report. Am J Orthod 75:291-300

4. Lines PA, Steinhauser EW (1974) Soft tissue changes in relationship to movement of hard structures in orthognathic surgery: a preliminary report. J Oral Surg 32:891

5. Schendel SA, Eisenfeld JH, Bell WH et al (1976) Superior repositioning of the maxilla: stability and soft tissue osseous relations. Am J Orthod 70:663

6. Schendel SA, Carlotti AE (1991) Nasal considerations in orthognathic surgery. Am J Orthod Dentofac Orthop 100:197-208

7. Jayaratne YSN, Zwahlen RA, Lo J, Cheung LK (2010) Facial soft tissue response to anterior segmental osteotomies: a systematic review. Int J Oral Maxillofac Surg 39:1050-1058

8. Park Je Uk, Hwang Young Sook et al (2008) Evaluation of the soft and hard tissue changes after anterior segmental osteotomy on the maxilla and mandible. J Oral Maxillfac Surg 66:98-103

9. Okudaira Mariko, Kawamoto Tatsuo, Ono Takashi (2008) Soft tissue changes in association with anterior maxillary osteotomy: a pilot study. Oral Maxillofac Surg 212:131-138

10. NataoSuda Kimie Ohyama, Fukada Kenji (2002) Three cases of anterior maxillary osteotomy under orotracheal intubation. Int $\mathrm{J}$ Adult Orthod Orthognath Surg 2002(17):273-282

11. Radney LJ, Jacobs JD (1981) Soft tissue changes associated with surgical total maxillary intrusion. Am J Orthod 80(2):191-212

12. Mansour Stephen, Burstone Charles (1983) An evaluation of soft tissue changes resulting from Le Fort I maxillary surgery. Am J Orthod 84(1):37-47

13. Freihofer HP (1976) The lip profile after correction of retromaxillism in cleft and non cleft patients. J Maxillofac Surg 4:136-141

14. Schendel Stephen A, Williamson Lewis W (1983) Muscle reorientation following repositioning of maxilla. J Oral Maxillofac Surg 41:235-240

15. Wu ZX, Zheng LW (2010) Subapical anterior maxillary segmental osteotomy: a modified surgical approach to treat maxillary protrusion. J Craniofac Surg 21(1):97-100 